\title{
Establishing a terrestrial proxy based on fluorescent dissolved organic matter from sediment pore water in the East China Sea
}

\author{
Minchun Li ${ }^{1,2}$, Wei XIE ${ }^{1,2 *}$, Penghui Li ${ }^{3}$, ChuANLUN \\ $\mathrm{ZHANG}^{3 *}$ \\ ${ }^{1}$ School of Marine Science, Sun Yat-sen University, Zhuhai, \\ 519082, China $(*$ correspondence: \\ xiewei9@mail.sysu.edu.cn) \\ ${ }^{2}$ Southern Marine Science and Engineering Guangdong \\ Laboratory (Zhuhai), Zhuhai, 519082, China \\ (*correspondence: xiewei9@mail.sysu.edu.cn) \\ ${ }^{3}$ Shenzhen Key Laboratory of Marine Archaea Geo-Omics, \\ Department of Ocean Science \& Engineering, Southern \\ University of Science and Technology, Shenzhen, 518055, \\ China (*correspondence: zhangcl@sustech.edu.cn)
}

Fluorescent dissolved organic matter (FDOM) is an important fraction of chromophoric dissolved organic matter (CDOM), which plays an important role in oceanic carbon cycle. In this study, surface sediment samples were collected from forty-two sites in the coastal region of the East China Sea (ECS) to examine the distributional patterns of CDOM. Three protein-like components $(\mathrm{C} 1, \mathrm{C} 4$ and $\mathrm{C} 5)$ and two humic-like components ( $\mathrm{C} 2$ and $\mathrm{C} 3$ ) of the $\mathrm{CDOM}$ were identified using fluorescence excitation-emission matrices parallel factor analysis (EEMs-PARAFAC). Spatially, the intensity of these five components generally increased from the coast to the ocean with protein-like components showing a more obvious trend, which suggested that all five components had autochthonous contribution. However, the $\mathrm{C} 2$ and $\mathrm{C} 3$ proportions significantly decreased from the coast to the ocean and profoundly correlated with the Branched and Isoprenoid Tetraether (BIT) index from corresponding solid fractions. We posit that part of the humic-like components from terrestrial organic matter in the sediments are released into the $\mathrm{C} 2$ and $\mathrm{C} 3$ pools in the pore water, which may be constrained by specific environmental conditions. Thus, the FDOM from the pore water can be integrated with BIT to validate the nature of FDOM and use it as a biomarker to reflect the terrestrial input of organic matter mediated by different biogeochemical processes in coastal oceans. 